\title{
Modulation of Dopaminergic and Glutamatergic Brain Function: PET Studies on Parkinsonian Rats
}

\author{
Daniela Pellegrino ${ }^{1}$, Francesca Cicchetti ${ }^{2,3}$, Xukui Wang ${ }^{1}$, Aijun $\mathrm{Zhu}^{1}$, Mexiang Yu$^{1}$, Martine Saint-Pierre ${ }^{2}$, and \\ Anna-Liisa Brownell ${ }^{1}$ \\ ${ }^{I}$ Experimental PET Laboratory, Department of Radiology, Massachusetts General Hospital, Harvard Medical School, Boston, \\ Massachusetts; ${ }^{2}$ Centre de Recherche en Neurosciences, CHUL, Québec, Canada; and ${ }^{3}$ Department de Médecine, Université Laval, \\ Québec, Canada
}

Degeneration of dopaminergic neurons of the substantia nigra pars compacta is a cardinal feature of Parkinson's disease (PD). Although uncertain, the pathology has been suggested to derive from a malfunction of the complex interaction between dopaminergic and metabotropic glutamate receptors (mGluRs). To further address this issue, we investigated the imaging profile and expression of dopamine $D_{2}$ receptors and mGluRs in a classic parkinsonian rodent model induced by the toxin 6-hydroxydopamine. Methods: Adult male Sprague-Dawley rats $(250-300 \mathrm{~g})$ received a stereotaxic injection of $8 \mu \mathrm{g} / 2 \mu \mathrm{L}$ of 6 -hydroxydopamine $(n=6)$ or saline solution $(n=4)$ in the right medial forebrain bundle. Small-animal PET was performed on all rats $4 \mathrm{wk}$ after the surgical procedure to assess dopamine transporter (DAT) status using ${ }^{11} \mathrm{C}-2 \beta$-carbomethoxy-3 $\beta-(4-$ fluorophenyl)-tropane (CFT), as well as dopamine $D_{2}$ receptor and mGluR $_{5}$ modulation using ${ }^{11} \mathrm{C}$-raclopride and $2-{ }^{11} \mathrm{C}$-methyl6-(2-phenylethynyl)-pyridine ( $\left.{ }^{11} \mathrm{C}-\mathrm{MPEP}\right)$, respectively. Behavioral studies were also conducted 6 wk after lesioning by D-amphetamine challenge. Immunohistochemistry and Western blotting were carried out at $8 \mathrm{wk}$ after lesioning to confirm dopamine fiber, neuronal loss, and level of striatal mGluR $_{5}$ expression. Results: PET images showed decreased ${ }^{11} \mathrm{C}-\mathrm{CFT}$ binding on the lesioned side, including the structures of the striatum, hippocampus, and cortex, compared with the contralateral intact side. Interestingly, dopamine $D_{2}$ receptors and mGluR $_{5}$ upregulation were observed in the right striatum, hippocampus, and cortex, using ${ }^{11} \mathrm{C}$-raclopride and ${ }^{11} \mathrm{C}-\mathrm{MPEP}$, respectively. A negative correlation was also found between the percentage change in mGluR $_{5}$ expression and DAT function. Finally, tyrosine hydroxylase immunoreactivity confirmed both dopamine fiber loss ( $t$ test, $P<0.01$ ) and neuronal loss ( $t$ test, $P<0.01$ ) on the lesioned side. These changes were accompanied by a strongly enhanced mGluR $_{5}$ expression in the right striatum of the lesioned side analyzed by Western plot. Conclusion: These findings support the existence of compensatory mechanisms in nigrostriatal dopamine degeneration and provide new insights that help further dissect some of the pathways underlying neurodegeneration. In addition, these results reconfirm that PET is a valuable tool for multilevel receptor studies, significantly contributing to the understanding of patho-

Received Nov. 14, 2006; revision accepted Mar. 20, 2007.

For correspondence or reprints contact: Anna-Liisa Brownell, PhD, Department of Radiology, Massachusetts General Hospital, Bartlett Hall, 504R, Boston, MA 02114.

E-mail: abrownell@partners.org

COPYRIGHT @ 2007 by the Society of Nuclear Medicine, Inc. genic mechanisms and ultimately opening new avenues in the study of neuroprotective approaches toward PD.

Key Words: neurology; PET; dopamine receptors; dopamine transporter; glutamate receptors; Parkinson's disease

J Nucl Med 2007; 48:1147-1153

DOI: 10.2967/jnumed.106.037796

$\mathbf{N}$ igrostriatal dopaminergic denervation, distinctive of Parkinson's disease (PD), has been suggested to result when a failure in the complex modulation of the neurochemical function of the basal ganglia causes increased glutamatergic activity in the subthalamic nucleus. Under normal conditions, the dopaminergic circuitry is involved in the control of locomotion and in the modulation of behaviors such as emotion and cognition (1-4). The striatum, a component of the basal ganglia, is a target for inhibitory dopaminergic inputs from the substantia nigra and the ventral segmental area, as well as from the hippocampus, periventricular thalamus, and amygdala $(5,6)$. Excitatory glutamatergic projections originate predominantly from prefrontal cortical areas and subthalamic structures, which in turn regulate the substantia nigra $(1,7,8)$. The dopaminergic and glutamatergic systems of the basal ganglia are thus reciprocally involved in a complex circuitry controlling motor behavior $(1,3)$. It has also been suggested that dopamine deficiency may be secondary to overactivity of the glutamatergic pathways, further contributing to the development of parkinsonian symptoms (9). Targeting the excessive glutamate release might therefore be a nondopamine approach to improving the symptoms of PD.

At the cellular level, several studies have demonstrated that both ionotropic and metabotropic glutamate receptors (mGluRs) are localized in the striatum $(10,11)$. Group I (mGluR 1 and mGluR $_{5}$ ) is G-protein-coupled membrane receptors localized mainly postsynaptically and expressed in striatonigral, striatopallidal projection neurons and in striatal interneurons. The subtypes $\mathrm{mGluR}_{1}$ and $\mathrm{mGluR}_{5}$ have been shown to activate phospholipase $\mathrm{C}$ and phosphoinositide hydrolysis and increase neuronal excitability 
(12). Stimulation of groups II (mGluR $R_{2}$ and $\left.\mathrm{mGluR}_{3}\right)$ and III $\left(\mathrm{mGluR}_{4}, \mathrm{mGluR}_{6}, \mathrm{mGluR}_{7}\right.$, and $\mathrm{mGluR}_{8}$ ) decreases adenylate cyclase activity, cAMP level, neuronal activity, and glutamate release $(12,13)$. Pharmacologic and biochemical findings have also confirmed that GluR $_{5}$ directly controls the phosphorylation state of transmembrane dopamine transporter (DAT) protein by decreasing the efficiency of reuptake/release of dopamine at the presynaptic terminals (14). MGluR antagonists may thus exhibit antiparkinsonian properties by blocking the receptors and inhibiting the induction and progression of glutamate-induced excitotoxic damage within the basal ganglia $(15,16)$. Several noncompetitive ionotropic-glutamate-receptor antagonists such as phencyclidine palmitate, MK-801, and CNS-1102 are used to block the action of glutamate receptors and have shown in vitro neuroprotective effects for excessive and inadequate uptake of synaptic glutamate (17). These ligands have been tested for neuroprotection in pathologic conditions such as stroke, brain injury, or neurodegenerative disorders such as PD, Alzheimer's disease, and Huntington's disease. In this context, in vivo imaging studies can provide crucial information that may help understanding receptor functions in normal and pathologic conditions.

PET, SPECT, and functional MRI have proven to be particularly valuable for in vivo imaging of nigrostriatal dopaminergic function in PD (18-20). The presynaptic terminals can be imaged using various radiolabeled ligands: ${ }^{18}$ F-fluorodopa (with PET), to evaluate uptake and conversion of fluoro-dopa into fluoro-dopamine by measuring the aromatic acid L-amino decarboxylase activity; ${ }^{11} \mathrm{C}$ dihydrotetrabenazine (with PET), to calculate the density of dopamine vesicles containing monoamines; cocaine analogs, such as ${ }^{123} \mathrm{I}-2 \beta$-carbomethyl-3 $\beta$-(4-iodophenyl)tropane (with SPECT) and ${ }^{11} \mathrm{C}-2 \beta$-carbomethoxy-3 $\beta$-(4fluorophenyl)-tropane (CFT) (with PET), to quantify DAT levels $(21,22)$; and ${ }^{11} \mathrm{C}$-raclopride (with PET), which competes with endogenous dopamine by binding to the dopamine $D_{2}$ receptors and thus can be used for indirect determination of dopamine release (23). The development of new pharmacologic probes for in vivo imaging of glutamate receptors is particularly important, to investigate neurochemical modulation of dopamine and glutamate receptors in different neurologic conditions $(24,25)$.

Thus far, several ${ }^{11} \mathrm{C}$-labeled pyridine analogs such as ${ }^{2-}{ }^{11} \mathrm{C}$-methyl-6-(2-phenylethynyl)-pyridine $\quad\left({ }^{11} \mathrm{C}-\mathrm{MPEP}\right)$, 2-[2-(3- ${ }^{11} \mathrm{C}-$ methoxyphenyl)-ethynyl]-pyridine, and 2-[2$\left(5-{ }^{11} \mathrm{C}\right.$-methoxypyridin-3-yl)-ethynyl]-pyridine have been developed and tested for in vivo imaging as specific antagonists of mGluRs (26). Previous PET studies performed on rodent models showed that these ligands rapidly accumulate and bind mainly within the striatum, cortical areas, and hippocampus 2-3 min after administration (26).

MPEP, a highly selective and noncompetitive antagonist of $\mathrm{mGluR}_{5}$, completely inhibits the decrement of dopamine uptake at presynaptic dopaminergic terminals by antago- nizing the in vivo effects of haloperidol on muscular rigidity $(14,16)$. Other in vitro studies using cortical rat cells support the neuroprotective effects of MPEP against glutamate-mediated excitotoxicity (27). Recently, Hamill et al. (28) have successfully used poly(ethylene-co-butylene naphthalene-2,6-dicarboxylate), which irreversibly binds to $\mathrm{mGluR}_{5}$, to perform in vivo imaging studies on rhesus monkeys.

The purpose of the present study was to investigate modulation of dopaminergic and $\mathrm{mGluR}_{5}$ function in 6-hydroxydopamine-lesioned rats using in vivo small-animal PET, with ultimate comparison to in vitro immunohistochemistry and Western blot analyses.

\section{MATERIALS AND METHODS}

\section{Experimental Design}

This study was performed on 10 male Sprague-Dawley rats (250-300 g; 8-10 wk old; Charles River Laboratories), cared for in accordance with the guidelines of the Committee of Animals of The Massachusetts General Hospital. The animals were divided into 2 groups: 6 rats that received stereotactically and unilaterally administered 6-hydroxydopamine ( $8 \mu \mathrm{g} / 2 \mu \mathrm{L}$, Sigma) in the right medial forebrain bundle, and 4 control rats that similarly received saline solution (29). Behavior was assessed in all lesioned and control rats 6 wk after the surgical procedure. Small-animal PET was performed $4 \mathrm{wk}$ after the lesioning in all rats to investigate modulation of dopaminergic and glutamatergic function within the basal ganglia. Endpoint immunohistochemical and Western blot analyses were performed to verify the extent of the lesions and the striatal $\mathrm{mGluR}_{5}$ expression, respectively.

\section{PET Ligands}

PET ligands were synthesized at the cyclotron laboratory at Massachusetts General Hospital. ${ }^{11} \mathrm{C}-\mathrm{CFT}$, a selective DAT ligand, was used to investigate dopaminergic brain function $(30) .{ }^{11} \mathrm{C}$ Raclopride was used to indirectly measure dopamine release (18), and ${ }^{11} \mathrm{C}$-MPEP, a selective $\mathrm{mGluR}_{5}$ antagonist, was used to investigate glutamatergic function (26).

\section{PET Studies}

Imaging studies were performed on six 6-hydroxydopaminelesioned and 4 control rats using a small-animal PET tomograph (microPET P4; Concord Microsystems). Anesthetized rats (1.5\%$2 \%$ isoflurane with $1 \mathrm{~L} / \mathrm{min} \mathrm{O}_{2}$ ) were positioned prone in the scanner with the head placed into a stereotactic holder built inhouse. To correct for attenuation, we first obtained a 10-min transmission scan using a rotating ${ }^{68} \mathrm{Ge} /{ }^{68} \mathrm{Ge}$ pin source. Radioligands (37-59.2 MBq, with a specific activity of $44.4 \pm 14.8 \mathrm{GBq} / \mu \mathrm{mol}$ for ${ }^{11} \mathrm{C}$-CFT, $20.35 \pm 5.18 \mathrm{GBq} / \mu \mathrm{mol}$ for ${ }^{11} \mathrm{C}$-raclopride, and $33.3 \pm 12.95 \mathrm{GBq} / \mu \mathrm{mol}$ for ${ }^{11} \mathrm{C}$-MPEP) were administered as a bolus through the tail vein. After administration of the radioligand, volumetric dynamic emission data were acquired for $60 \mathrm{~min}$ for the entire brain. The imaging studies with 3 different ligands were conducted within 2-3 d. A study of DAT function using ${ }^{11} \mathrm{C}$ CFT was first performed to determine lesion size and was then followed by $\mathrm{mGluR}_{5}$ function and dopamine $\mathrm{D}_{2}$ receptor studies the next and the third day, respectively. Whenever 2 imaging studies were performed in the same imaging session, the ${ }^{11} \mathrm{C}$ MPEP study was performed first because of the fast washout of ligand from regions of interest and was followed by the ${ }^{11} \mathrm{C}-\mathrm{CFT}$ 
study $2 \mathrm{~h}$ later. Finally, the study with ${ }^{11} \mathrm{C}$-raclopride was routinely performed the next day.

The dynamic volumetric data, acquired in list mode, were sequenced to histograms using the following framing: $20 \times 15 \mathrm{~s}$, $25 \times 60 \mathrm{~s}$, and $10 \times 180 \mathrm{~s}$. After correction for uniformity, attenuation, and decay, the imaging data were reconstructed using a filtered backprojection, a ramp filter (cutoff, 0.5), and the software provided by the manufacturer (ASIPro, version 6.0; Concord Microsystems). The regions of interest, that is, cerebellum, left and right striatum, hippocampus, and cortex, were drawn onto coronal slices according to the brain outlines as derived from the rat brain atlas (31).

The binding potential for each radioligand in different brain areas was calculated by a modified graphical method developed by Logan et al. (32), using the cerebellum as the reference tissue. Percentage changes between left and right brain areas were calculated using the unlesioned left side as a reference area.

\section{Behavioral Studies}

Evaluation of rotational behavioral changes was done under a D-amphetamine challenge $2 \mathrm{wk}$ after PET imaging to avoid temporary alterations of dopaminergic function feasibly induced by D-amphetamine (33). D-amphetamine is an indirect agonist of dopamine receptors that introduces asymmetric rotation in rats that have received 6-hydroxydopamine unilaterally. For this procedure, rats were allowed to habituate for $30 \mathrm{~min}$ in a transparent cylinder (diameter, $39.5 \mathrm{~cm})$. After a single injection of D-amphetamine (5 $\mathrm{mg} / \mathrm{kg}$ intraperitoneally; Sigma), the rotational locomotor activity was video-recorded for 90 min (Digital Handy Cam DCR-TRV30 NTSC; Sony). The net left turns were calculated and used as a measure of parkinsonian syndromes (29).

\section{Immunohistochemistry}

Eight weeks after lesioning, 3 PD rats and 2 controls were deeply anesthetized ( $80 \mathrm{mg}$ of ketamine per kilogram, intraperitoneally) and sacrificed via intracardiac perfusion with $200 \mathrm{~mL}$ of cold $0.9 \%$ saline solution followed by $200 \mathrm{~mL}$ of ice cold $4 \%$ paraformaldehyde (Sigma) in phosphate-buffered saline (PBS, $0.1 \mathrm{M}, \mathrm{pH} 7.4$; Sigma). The brains were removed, fixed for $12 \mathrm{~h}$ in $4 \%$ paraformaldehyde, and transferred for $24 \mathrm{~h}$ into $20 \%$ sucrose/ PBS for cryoprotection. Tissues were sliced into $35-\mu \mathrm{m}$ coronal sections using a freezing microtome (SM 2000R; Leica), serially collected in antifreeze solution, and subsequently retrieved for each experiment. Free-floating sections were washed in PBS and placed in $3 \%$ peroxide for $30 \mathrm{~min}$ at room temperature. Slices were transferred in $0.1 \mathrm{M}$ PBS for several washes and preincubated in a $0.1 \mathrm{M}$ PBS solution containing 5\% normal goat serum (Wisent Inc.) and 0.1\% Triton X-100 (Sigma) for $30 \mathrm{~min}$ at room temperature. Sections were then incubated overnight at $4^{\circ} \mathrm{C}$ in rabbit anti-tyrosine hydroxylase (TH) (1:5,000; Pel-Freeze). After incubation with the primary antibody, tissue sections were washed in PBS and incubated for $1 \mathrm{~h}$ at room temperature in a PBS solution containing 5\% normal goat serum, $0.1 \%$ Triton $\mathrm{X}-100$, and biotinylated goat antirabbit antibody (1:1,500 diluted; Vector Lab). After several rinses in PBS, the sections were placed in a solution containing avidin-biotin peroxidase complex (ABC elite kit; Vector Lab) for $1 \mathrm{~h}$ at room temperature and subsequently washed twice in PBS. The bound antibodies were visualized by placing the sections in Tris buffer solution containing $0.05 \% 3$, 3 '-diaminobenzidine (Sigma) and $0.01 \%$ hydrogen peroxide, $30 \%$, at room temperature (Sigma). The reaction was stopped by washing in $0.05 \mathrm{M}$ Tris buffer. TH sections were dehydrated and coverslipped using DPX mounting medium (Electron Microscopy Science). A negative control was included in the experiments by incubating sections in PBS and omitting the primary antibody from the incubation medium.

\section{Stereologic Counts of TH Neuronal Loss}

The loss of dopaminergic neurons was determined by counting the average of TH-immunoreactive neurons in the 3 substantia nigra pars compacta sections at high magnification $(20 \times)$ under bright-field illumination (E800 Nikon microscope; Nikon Instruments). The cell count was performed in a masked fashion by 2 independent investigators. Analysis of TH-immunoreactive cells was restricted to the substantia nigra pars compacta and thus excluded the ventral tegmental area.

\section{Densitometric Evaluation of Striatal TH Fibers}

Digitized brain images of the striatum were captured using a charge-coupled-device camera (XC-77; Sony). The density of striatal dopaminergic fibers was analyzed using Image J software (version 1.63; National Institutes of Health). The average labeling for each area was calculated from 4 adjacent brain sections of the same animal at the level of the anterior commissure. Striatal images converted to gray scale were then delineated, and the intensity of staining was assessed for the entire region of 4 sections and subsequently averaged for each animal. Background intensities taken from the corpus callosum devoid of TH staining were subtracted from every measurement.

Statistical analyses were performed using the unpaired Student $t$ test on StatView software (SAS institute). Data derived from the striatum and substantia nigra were expressed as mean values \pm SD.

\section{Western Blot Analysis}

Western blot analysis of mGluR $_{5}$ was performed $8 \mathrm{wk}$ after lesioning. Three lesioned rats and 2 controls were sacrificed by cardiac puncture, and the brains were collected.

Striata were dissected and homogenized in $10 \mathrm{w} / \mathrm{v}$ ice-cold lysis buffer $(50 \mathrm{mM}$ Tris- $\mathrm{HCl}, \mathrm{pH} 7.4 ; 1 \mathrm{mM}$ ethylenediamine tetraacetic acid; $150 \mathrm{mM} \mathrm{NaCl} ; 0.1 \%$ sodium dodecylsulfate; $0.5 \%$ deoxycholic acid; 1\% (octylphenoxy)polyethoxyethanol [Igepal CA-630; Rhone-Poulenc AG Co.]; $1 \%$ protease inhibitor cocktail [Sigma]). Homogenates were centrifuged for $20 \mathrm{~min}$ at $10,000 \mathrm{rpm}$ at $4^{\circ} \mathrm{C}$, and total protein concentration was determined by absorbance spectroscopy (Benchmark Plus; BioRad) with bovine serum albumin as standard protein (BCA Protein Assay Kit; Pierce).

For protein separation, supernatants (30 $\mu \mathrm{g}$ of total proteins) were added 1:1 to $3 \times$ sample buffer and boiled for $5 \mathrm{~min}$. Proteins were separated using 4\%-12\% Tris-glycine polyacrylamide gel electrophoresis precast gels (Bio-Rad) and electro-blotted onto nitrocellulose membrane (NitroPure, $0.45 \mu \mathrm{m}$; GE Osmonics Labstore) for $1 \mathrm{~h}$ at $100 \mathrm{~V}$ at $4^{\circ} \mathrm{C}$. Polyacrylamide gel residues were washed off the membranes using TBS-T buffer $(100 \mathrm{mM}$ Tris- $\mathrm{HCl}, 0.9 \% \mathrm{NaCl}, 0.1 \%$ polysorbate $20, \mathrm{pH} 7.4)$ before blocking unspecific sites overnight at $4^{\circ} \mathrm{C}$ in $8 \%$ nonfat dry milk in TBS-T. Membranes were then incubated for $1 \mathrm{~h}$ at room temperature with primary antibody against $\mathrm{mGluR}_{5}$ (antimGluR $_{5}$, dilution of 1:2,000 in TBS-T; Chemicon).

Blots were incubated for $1 \mathrm{~h}$ at room temperature in horseradish peroxidase-conjugated secondary antibody in TBS-T $(1: 10,000$ enterochromaffin-like antirabbit IgG; Amersham). Finally, blots were washed $3 \times 10 \mathrm{~min}$ in TBS-T and developed using the 


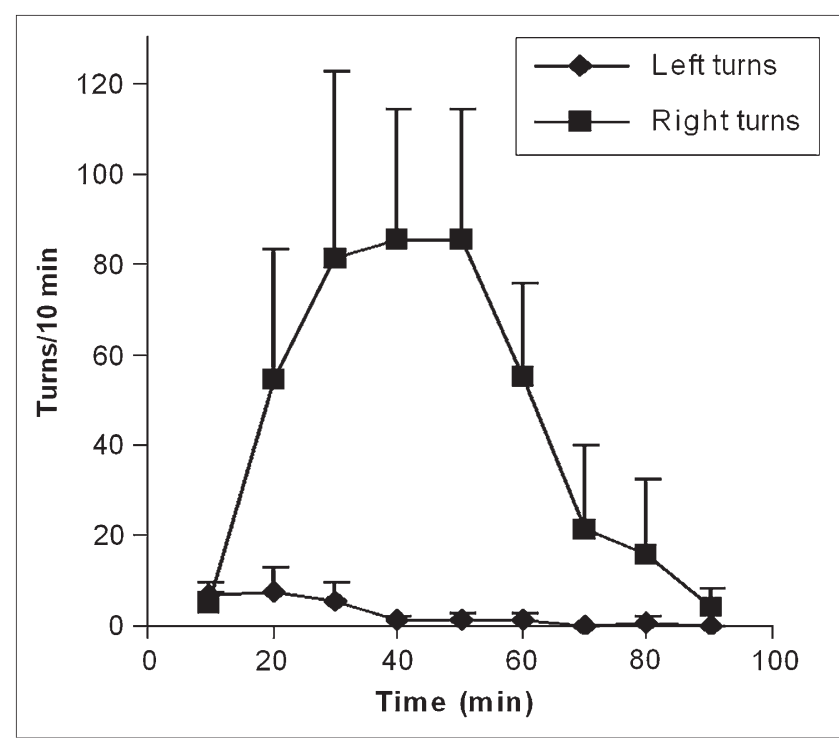

FIGURE 1. Number (mean $\pm \mathrm{SE}, n=6$ ) of left (contralateral) and right (ipsilateral) turns per 10 min registered 90 min after D-amphetamine administration (5 $\mathrm{mg} / \mathrm{kg}$ intraperitoneally). Rotational behavior was investigated 6 wk after unilateral administration of 6-hydroxydopamine into right medial forebrain bundle.

enhanced chemiluminescence procedure (SuperSignal West Pico Chemiluminescent Substrate; Pierce) followed by exposure to Kodak scientific imaging film for $10 \mathrm{~min}$ (Eastman Kodak). Immunoreactivity levels of $\mathrm{mGluR}_{5}$ were quantified by scanning the x-ray film. The density of receptors was evaluated by using Image $\mathbf{J}$ software.

\section{RESULTS}

\section{Imaging and Behavioral Correlations}

Six weeks after 6-hydroxydopamine injection, the scores greater than 5 full turns/min in lesioned animals demonstrate a reduced dopamine release within the injected site, compared with the contralateral side (Fig. 1). Net ipsilateral rotation induced by D-amphetamine challenge showed an exponential correlation to the percentage decrease in ${ }^{11} \mathrm{C}$ CFT binding potential in the right lesioned striatum. We established that the net ipsilateral rotations in 90 min can be expressed by the equation $111.1\left[\exp { }^{(0.045 \times \Delta(D A T)}-1\right]$ (at $95 \%$ confidence levels, where $\Delta$ is the percentage decrease in ${ }^{11} \mathrm{C}-\mathrm{CFT}$ binding potential between the left and right striata). Control rats did not exhibit any rotational behavior under D-amphetamine influence.

\section{Imaging of Dopaminergic and Glutamatergic Function}

In vivo imaging studies of DAT using ${ }^{11} \mathrm{C}-\mathrm{CFT}$ showed a significant decrease in accumulation in the right striatum suggesting a degeneration of dopaminergic function induced by 6-hydroxydopamine administration (Fig. 2). Time-activity curves of ${ }^{11} \mathrm{C}-\mathrm{CFT}$ accumulation showed fast washout in the right striatum. ${ }^{11} \mathrm{C}-\mathrm{CFT}$ binding potential was significantly decreased on the lesioned side, compared with the intact left striatum $(-33.0 \% \pm 14.3 \%$, with a range of $15 \%-49 \%, P<0.005)$. The hippocampal area showed a decreased ${ }^{11} \mathrm{C}-\mathrm{CFT}$ binding potential $(-4.2 \% \pm$ $2.8 \%, P<0.01)$, whereas the binding potential was unchanged in the cortex $(-0.2 \% \pm 7.4 \%, P>0.05)$.
FIGURE 2. Accumulation of ${ }^{11} \mathrm{C}-\mathrm{CFT}$, ${ }^{11} \mathrm{C}$-raclopride, and ${ }^{11} \mathrm{C}-\mathrm{MPEP}$ in 6-hydroxydopamine-lesioned rat brain. Distribution of ${ }^{11} \mathrm{C}-\mathrm{CFT}$ is illustrated at 20-30 $\mathrm{min},{ }^{11} \mathrm{C}$-raclopride at $15-25 \mathrm{~min}$, and ${ }^{11} \mathrm{C}-M P E P$ at $7-15 \mathrm{~min}$ after ligand administration. Images on left show decreased accumulation of ${ }^{11} \mathrm{C}-\mathrm{CFT}$, enhanced accumulation of ${ }^{11} \mathrm{C}$-raclopride, and enhanced accumulation of ${ }^{11} \mathrm{C}$ MPEP in right striatum compared with left control striatum. Sections are 1.25 $\mathrm{mm}$ thick. Plots on right show rate of radiotracer accumulation in striatum (percentage injected dose [\%id] per cubic centimeter) over time. Note fast washout of ${ }^{11} \mathrm{C}-\mathrm{CFT}$ from right striatum and enhanced accumulation of ${ }^{11} \mathrm{C}$-raclopride and ${ }^{11} \mathrm{C}$-MPEP in same brain area. $\mathrm{Hg}=$ harderian glands; $\mathrm{L}=$ left striatum; $\mathrm{R}=$ right striatum; $\mathrm{Rac}=$ raclopride.

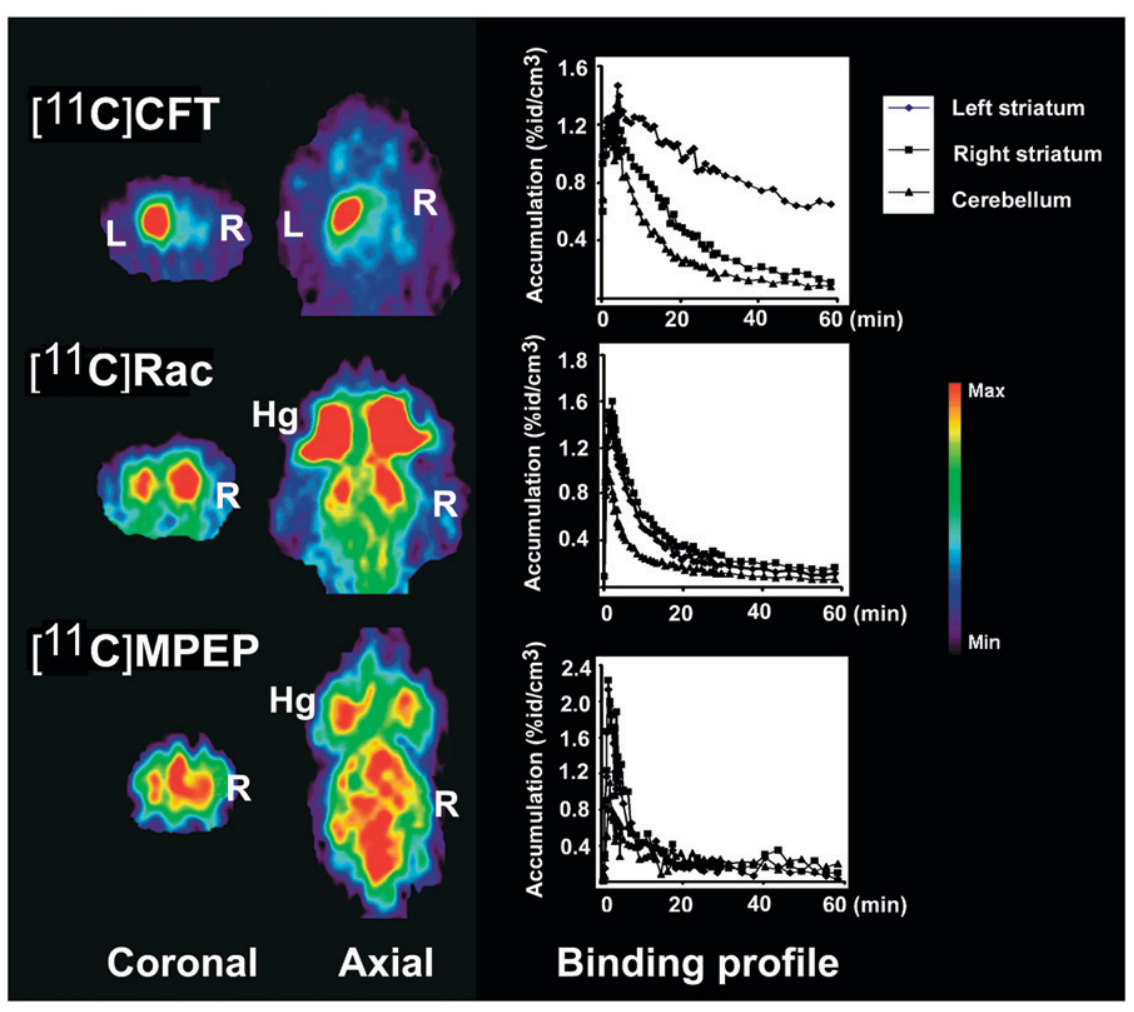




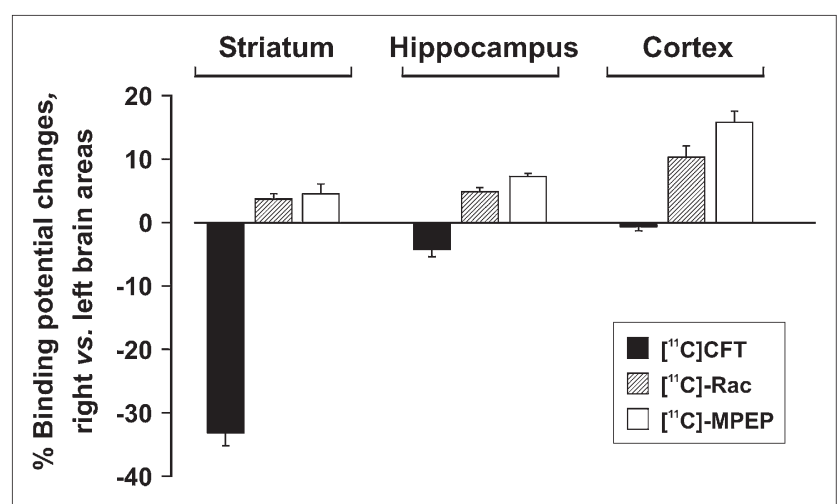

FIGURE 3. Percentage binding potential changes in ${ }^{11} \mathrm{C}-\mathrm{CFT}$, ${ }^{11} \mathrm{C}$-raclopride, and ${ }^{11} \mathrm{C}$-MPEP between right and left striatum, hippocampus, and cortex. ${ }^{11} \mathrm{C}-\mathrm{CFT}$ binding potential shows significant decrease in right striatum $(P<0.005)$ and in hippocampal areas $(P<0.01)$ and no significance in right cortex. Both ${ }^{11} \mathrm{C}$-raclopride and ${ }^{11} \mathrm{C}$-MPEP binding potentials are significantly increased in right striatum $(P<0.05)$, hippocampus, and cortex $(P<0.01)$. Values were calculated as \% (binding potential [right] - binding potential [left])/binding potential (left) and expressed as mean $\pm \mathrm{SE} . \mathrm{Rac}=$ raclopride.

Time-activity curves of ${ }^{11} \mathrm{C}$-raclopride showed $10 \%-$ $15 \%$ higher accumulation and delayed washout in the right striatum (Fig. 2). The ${ }^{11} \mathrm{C}$-raclopride binding potential showed upregulation of dopamine $\mathrm{D}_{2}$ receptor function in the right striatum $(3.6 \% \pm 1.6 \%, P<0.05)$, hippocampus $(4.8 \% \pm 3.16 \%, P<0.01)$, and cortex $(10.3 \% \pm 5.06 \%$, $P<0.01$ ) (Fig. 3). In parallel with this observation, timeactivity curves of ${ }^{11} \mathrm{C}$-MPEP were enhanced in the right striatum of lesioned animals, compared with the untreated counterparts $(4.5 \% \pm 0.9 \%, P<0.01)$, indicating enhanced $\mathrm{mGluR}_{5}$ function. Enhanced binding potential was also observed in the hippocampus $(7.1 \% \pm 4.8 \%, P<$ $0.01)$ and cortex $(15.6 \% \pm 12.1 \%, P<0.01)$ (Fig. 3). A negative correlation was found between the percentage change $(\Delta)$ in mGluR $_{5}$ expression and DAT function: For the striatum, $\Delta\left(\mathrm{mGluR}_{5}\right)=-0.18 \Delta(\mathrm{DAT}), r=0.6, P>$ 0.05 . Significance was not obtained because of the small sample size and large variation between the animals, but the trend $\Delta\left(\mathrm{mGluR}_{5}\right)$ or $\Delta$ (raclopride $)=-\mathrm{k} \Delta(\mathrm{DAT})$ was obvious for all investigated brain areas.
In the control animals, ${ }^{11} \mathrm{C}-\mathrm{CFT},{ }^{11} \mathrm{C}$-raclopride, and ${ }^{11} \mathrm{C}$ MPEP accumulation did not show any differences between the right and left brain areas.

\section{Postmortem Histologic Confirmation of Dopamine Alteration}

To further investigate and demonstrate the dopamine depletion, 3 lesioned rats underwent postmortem histologic analyses. Lesions were readily detectable in the right medial forebrain bundle at the site of 6-hydroxydopamine inoculation in all examined animals There was a loss of TH-immunoreactive fibers $(P<0.01$, Fig. 4A), compared with the contralateral nonlesioned side, and a decrease in the number of nigral TH-immunoreactive neurons $(P<$ 0.01, Fig. 4B), confirming successful 6-hydroxydopamine lesioning (Figs. 4 and 5).

\section{Postmortem Evidence of mGluR $_{5}$ Upregulation}

Eight weeks after 6-hydroxydopamine lesioning, both injected and contralateral normal tissues were analyzed by Western blot with an mGluR $_{5}$ monoclonal antibody. Both tissues showed a single band, corresponding to the molecular mass of $\mathrm{mGluR}_{5}$ receptor $(128 \mathrm{kDa})$. As clearly visible on the immunoblot, the optical density readout confirmed a strong upregulation of $\mathrm{mGluR}_{5}$ in the right striatum (lesioned side) in all PD rats examined (Fig. 6). In contrast, no difference in $\mathrm{mGluR}_{5}$ expression was observed between the left and right striatum of the control animals (Fig. 6).

\section{DISCUSSION}

It has been extensively demonstrated that the subthalamic nucleus in PD is overactive as a result of nigrostriatal degeneration. This feature has been thought to play an important role in the pathogenesis of PD through the enhancement of glutamate in the indirect pathway (34). Metabotropic glutamate transmission is enhanced in the basal ganglia circuit, particularly in the striatum, where it interacts with the dopamine system to control movement. It is also well documented that an excessive activation of mGluR $_{5}$ has been implicated in several neurodegenerative disorders such as anxiety, depression, schizophrenia, and drug addiction (7).

Using ${ }^{11} \mathrm{C}$-CFT as a specific biomarker for DAT at the presynaptic terminal, we observed a decreased ${ }^{11} \mathrm{C}$-CFT

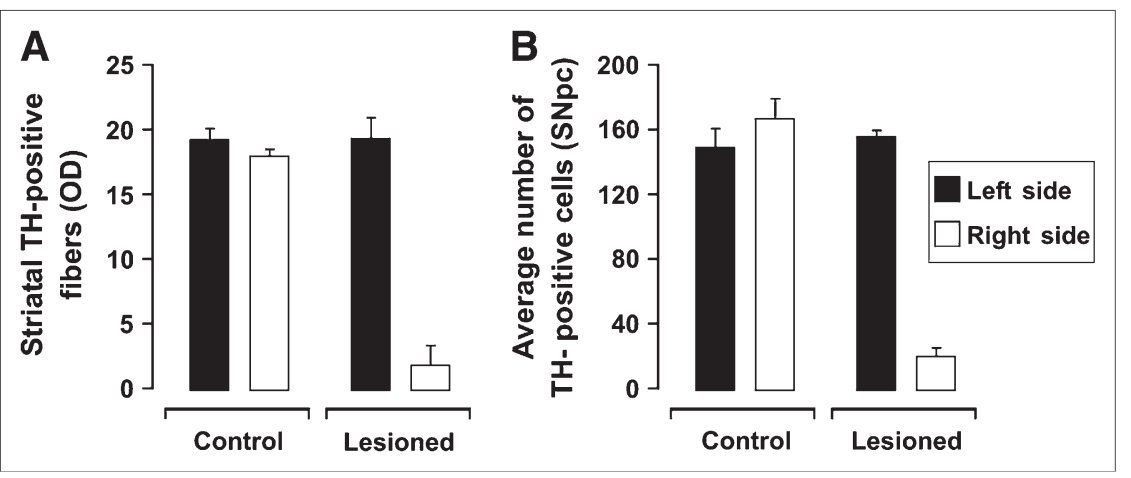

FIGURE 4. TH-immunoreactive fibers were significantly decreased in lesioned striatum $(P<0.01)(\mathrm{A})$, and $\mathrm{TH}$ immune staining in substantia nigra of lesioned animal showed significant loss of $\mathrm{TH}$ expressing neurons in substantia nigra pars compacta (SNpc), whereas ventral tegmental area was relatively unaltered $(P<0.01)(\mathrm{B})$. 
FIGURE 5. Microscopic 35- $\mu \mathrm{m}$ coronal sections of $\mathrm{TH}$-stained striatum and substantia nigra pars compacta samples collected 8 wk after 6-hydroxydopamine administration. No differences in $\mathrm{TH}$ immunoreactivity between left and right striatum (A) or left and right substantia nigra pars compacta $(C)$ of control rat brain were observed. TH immunoreactivity of 6-hydroxydopamine-administered rat brain shows significant loss of $\mathrm{TH}$ immunoreactive fibers on lesioned striatum and substantia nigra pars compacta ( $B$ and $D)$. Neurons were counted under a bright field (20×, E800; Nikon). Scale bar $=1 \mathrm{~cm}$.
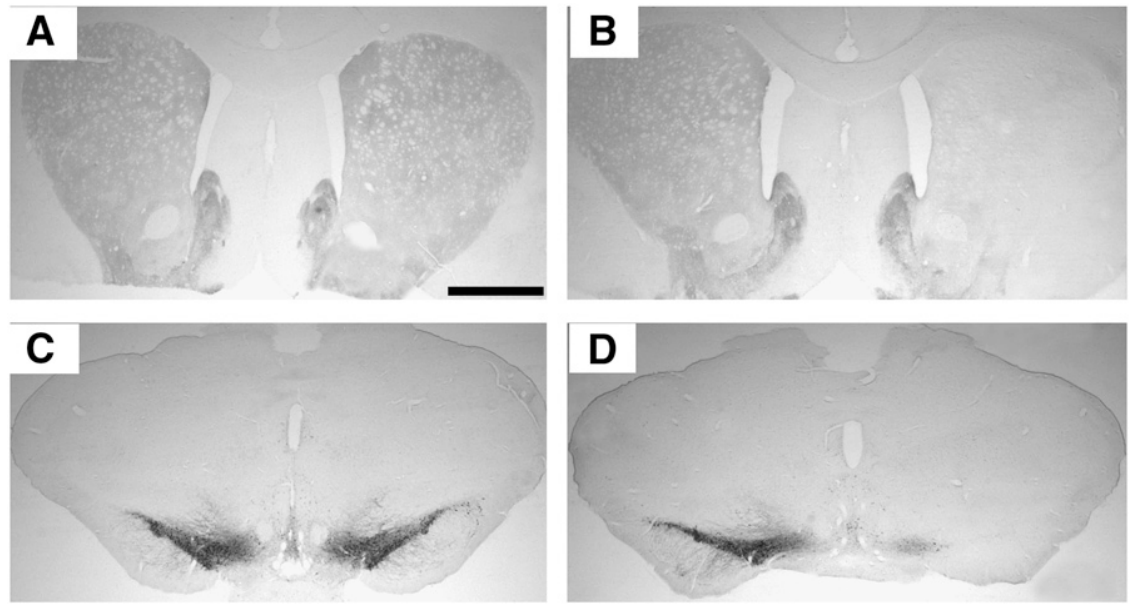

binding potential in the right striatum, compared with the intact left side. The decreased binding potential observed in the basal ganglia indicates loss of DAT function in the 6-hydroxydopamine-induced neurotoxicity model, and this decrease was confirmed by endpoint immunohistochemical studies. The DAT decrease in the lesioned striatum was exponentially proportional to the increased contralateral turns/min observed under D-amphetamine challenge. In fact, the rats showing the least ipsilateral rotations also showed partial denervation of striatal dopaminergic terminals as revealed by TH immunohistochemistry. The decrease in DAT also correlates with the increased uptake of ${ }^{11} \mathrm{C}$-MPEP in the right striatum and upregulation of $\mathrm{mGluR}_{5}$ expression as demonstrated by Western blot analysis. Similarly, Page et al. (14) showed that $\mathrm{mGluR}_{5}$ controls the decrease in DAT activity through activation of protein kinase-C and calcium calmodulin-dependent protein kinase II.

We consistently observed an enhanced expression of mGluR $_{5}$ and dopamine $\mathrm{D}_{2}$ receptors in the striatum, hippocampus, and cortex on the lesioned side of the brain. However, significance was not achieved in correlation analysis because of the small sample size and large variation between the animals, but the trend $\Delta\left(\mathrm{mGluR}_{5}\right)$ or $\Delta($ raclopride $)=-\mathrm{k} \Delta(\mathrm{DAT})$ is obvious for all investigated brain areas.

Enhancement of dopamine $\mathrm{D}_{2}$ receptor expression is an indication of functional hypersensitivity of dopamine re-

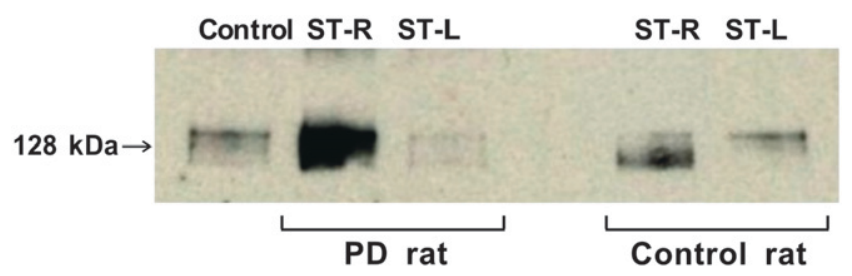

FIGURE 6. Immunoblotting analysis of $\mathrm{mGluR}_{5}$ protein in striata samples collected $8 \mathrm{wk}$ after 6-hydroxydopamine administration. Densitometric analysis of PD rat and control striatum shows presence of a single band at $128 \mathrm{kDa}$, corresponding to molecular mass of mGluR $_{5}$. ST-L $=$ left striatum; ST-R = right striatum. ceptors at the postsynaptic terminal. Several studies on progressive MPTP-lesioned primate PD models demonstrated that both an increase in $\mathrm{D}_{2}$ receptor binding and a breakdown of dopamine homeostasis are fundamental features of the early presymptomatic phase of PD (35).

A high specific activity of the radioligand is one of the first prerequisites for receptor studies. Typically, radiolabeling with ${ }^{11} \mathrm{C}$-methyl iodide produces picomolar amounts of hot compound, with a hot-to-cold compound ratio of 1:10,000. Of the 3 radioligands used, ${ }^{11} \mathrm{C}$-raclopride had the lowest specific activity; thus, if the maximum dopamine $\mathrm{D}_{2}$ receptor binding sites is $60 \mathrm{nmol}$, the cold compound will occupy about $3 \%$ of the binding sites. The radiotracers used for this study are on the order of picomoles; it is therefore conceivable that such a tiny amount does not affect system homeostasis. The significant increase in $\mathrm{mGluR}_{5}$ function evidenced by ${ }^{11} \mathrm{C}$-MPEP in lesioned striatum, hippocampus, and cortex indicates that modulation of glutamate receptors may play a functional role in rotational behavior. Although the metabolic pathways of $\mathrm{mGluR}_{5}$ upregulation are still unknown, in vitro $\mathrm{mGluR}_{5}$ findings paralleled the in vivo imaging results and suggest a functional activation of $\mathrm{mGluR}_{5}$. The inverse linear correlation between DAT and glutamatergic functions also suggests that an antagonist at the $\mathrm{mGluR}_{5}$ might play a role in dopamine neuromodulation. These data are consistent with the in vivo data published by Vernon et al. (36) demonstrating the protective effects of $\mathrm{mGluR}_{5}$ ligands in PD rats.

\section{CONCLUSION}

This work investigated the modulation of dopaminergic and glutamatergic function using in vivo PET imaging in a PD rat model induced by administration of 6-hydroxydopamine into the right medial forebrain bundle, creating stable, clearly manifested dopaminergic degeneration in $4 \mathrm{wk}$. Our data support the use of small-animal PET to investigate in vivo glutamatergic and dopaminergic function in 6-hydroxydopamine-lesioned rat models. In addition, our 
data demonstrate that metabotropic glutamatergic and dopaminergic function are interdependent and act synergistically in movement control. Therefore, the use of mGluR antagonists may potentially be neuroprotective in PD-like dopaminergic degeneration.

\section{ACKNOWLEDGMENTS}

This work was supported by grant EB-001850 from the NIH-NIBIB and by a Cassen Fellowship from the American Nuclear Medicine Society. We thank William Bucelewicz and David Lee at Massachusetts General Hospital for providing radioisotopes, and Deborah Schuback at the Breakefield Laboratory at Massachusetts General Hospital for technical support.

\section{REFERENCES}

1. Carlsson ML, Carlsson A. Interactions between glutamatergic and monoaminergic systems within the basal ganglia: implications for schizophrenia and Parkinson's disease. Trends Neurosci. 1990;13:272-276.

2. Kotter R. Postsynaptic integration of glutamatergic and dopaminergic signals in the striatum. Prog Neurobiol. 1994;44:163-196.

3. Greengard P, Allen PB, Nairn AC. Beyond the dopamine receptor: the DARPP32/protein phosphatase-1 cascade. Neuron. 1999;23:435-447.

4. Robbins TW. Chemical neuromodulation of frontal-executive functions in humans and other animals. Exp Brain Res. 2000;133:130-138.

5. Parent A, Hazrati LN. Functional anatomy of the basal ganglia. I. The cortico-basal ganglia-thalamo-cortical loop. Brain Res Brain Res Rev. 1995;20:91-127.

6. Parent A, Hazrati LN. Functional anatomy of the basal ganglia. II. The place of subthalamic nucleus and external pallidum in basal ganglia circuitry. Brain Res Brain Res Rev. 1995;20:128-154.

7. Phillipson OT, Griffiths AC. The topographic order of inputs to nucleus accumbens in the rat. Neuroscience. 1985;16:275-296.

8. Graybiel AM. Neurotransmitters and neuromodulators in the basal ganglia. Trends Neurosci. 1990;13:244-254.

9. Klockgether T, Tursky L. Excitatory amino acids and the basal ganglia: implications for the therapy of Parkinson's disease. Trends Neurosci. 1989;12:285-286.

10. Ohishi H, Shigemoto R, Nakanishi S, Mizuno N. Distribution of the messenger RNA for a metabotropic glutamate receptor, mGluR2, in the central nervous system of the rat. Neuroscience. 1993;53:1009-1018.

11. Testa CM, Standaert DG, Young AB, Penney JB Jr. Metabotropic glutamate receptor mRNA expression in the basal ganglia of the rat. J Neurosci. 1994; 14:3005-3018.

12. Pin JP, Duvoisin R. Neurotransmitter receptors-1: the metabotropic glutamate receptors—structure and function. Neuropharmacology. 1995;34:1-26.

13. Tallaksen-Greene SJ, Kaatz WK, Romano C, Albin RL. Localization of mGluR1 alike immunoreactivity and $\mathrm{m}$ GluR5-like immunoreactivity in identified population of striatal neurons. Brain Res. 1998;780:210-217.

14. Page G, Peeters M, Najimi M, Maloteaux JM, Hermans E. Modulation of the neuronal dopamine transporter activity by the metabotropic glutamate receptor mGlur5 in rat striatal synaptosomes through phosphorylation mediated process. J Neurochem. 2001;76:1282-1290.
15. Marek G. Metabotropic glutamate $2 / 3$ receptors as drug targets. Curr Opin Pharmacol. 2004;4:18-22.

16. Ossowska K, Konieczny J, Wolfarth S, Pilc A. MTEP, a new selective antagonist of the metabotropic glutamate receptor subtype 5 (mGluR5), produces antiparkinsonian-like effects in rats. Neuropharmacology. 2005;49:447-455.

17. Choi DW. Calcium-mediated neurotoxicity: relationship to specific channel types and role in ischemic damage. Trends Neurosci. 1988;11:465-469.

18. Brownell AL, Canales K, Chen YI, et al. Mapping of brain function after MPTPinduced neurotoxicity in a primate Parkinson's disease model. Neuroimage. 2003;20:1064-1075.

19. Jenkins BG, Sanchez-Pernaute R, Brownell AL, Chen YC, Isacson O. Mapping dopamine function in primates using pharmacologic magnetic resonance imaging. J Neurosci. 2004;24:9553-9560.

20. Eckert T, Eidelberg D. Neuroimaging and therapeutics in movement disorders. NeuroRx. 2005;2:361-371.

21. Brooks DJ. Positron emission tomography and single-photon emission computed tomography in central nervous system drug development. NeuroRx. 2005;2:226-236.

22. Ravina B, Eidelberg D, Ahlskog JE, et al. The role of radiotracer imaging in Parkinson disease. Neurology. 2005;64:208-215.

23. Farde L, Hall H, Ehrin E, Sedvall G. Quantitative analysis of D2 dopamine receptor binding in the living human brain by PET. Science. 1986;231:258-261.

24. Blin J, Denis A, Yamaguchi T, MacKenzie E, Baron J. PET studies of $\left[{ }^{18}\right.$ F $]$ methyl-MK-801, a potential NMDA receptor complex radioligand. Neurosci Lett. 1991;121:183-186.

25. Piccini $\mathrm{P}$, Brooks DJ, Bjorklund A, et al. Dopamine release from nigral transplants visualized in vivo in a Parkinson's patient. Nat Neurosci. 1999;2: 1137-1140.

26. Yu M, Tueckmantel W, Wang X, et al. Methoxyphenylethynyl, methoxypyridylethynyl and phenylethynyl derivatives of pyridine: synthesis, radiolabeling and evaluation of new PET ligands for metabotropic glutamate subtype 5 receptors. Nucl Med Biol. 2005;32:631-640.

27. O'Leary DM, Movsesyan V, Vicini S, Faden AI. Selective mGluR5 antagonist MPEP and SIB-1893 decrease NMDA or glutamate-mediated neuronal toxicity through actions that reflect NMDA receptor antagonism. Br J Pharmacol. 2000; 131:1429-1437.

28. Hamill TG, Krause S, Ryan C, et al. Synthesis, characterization, and first successful monkey imaging studies of metabotropic glutamate receptor subtype 5 (mGluR5) PET radiotracers. Synapse. 2005;56:205-216.

29. Brownell AL, Livni E, Galpern W, Isacson O. In vivo PET imaging in rat of dopamine terminals reveals functional neural transplant. Ann Neurol. 1998;43:387-390.

30. Brownell AL, Elmaleh DR, Meltzer PC, et al. Cocaine congeners as PET imaging probes for dopamine terminals. J Nucl Med. 1996;37:1186-1192.

31. Paxinos G, Watson C. The Rat Brain in Stereotaxic Coordinates. 4th ed. San Diego, CA: Academic Press; 2002.

32. Logan J, Fowler JS, Volkow ND, Wang GJ, Ding YS, Alexoff DL. Distribution volume ratios without blood sampling from graphical analysis of PET data. J Cereb Blood Flow Metab. 1996;16:834-840.

33. Olds ME, Jacques DB, Kopyov O. Relation between rotation in the 6-OHDA lesioned rat and dopamine loss in striatal and substantia nigra subregions. Synapse. 2006;59:532-544.

34. Rodriguez MC, Obeso JA, Olanow CW. Subthalamic nucleus-mediated excitotoxicity in Parkinson's disease: a target for neuroprotection. Ann Neurol. 1998;44(suppl):S175-S188.

35. Bezard E, Gross CE, Brotchie JM. Presymptomatic compensation in Parkinson's disease is not dopamine-mediated. Trends Neurosci. 2004;26:215-221.

36. Vernon AC, Palmer S, Datla KP, et al. Neuroprotective effects of metabotropic glutamate receptor ligands in a 6-hydroxydopamine rodent model of Parkinson's disease. Eur J Neurosci. 2005;22:1799-1806. 